\title{
Impact of COVID-19 on neurological patients attending a botulinum toxin service
}

\author{
Roberto Erro ${ }^{1}$ (D) $\cdot$ Sara Scannapieco ${ }^{1} \cdot$ Maria Russo $^{1} \cdot$ Marina Picillo $^{1} \cdot$ Paolo Barone $^{1}$
}

Received: 12 October 2020 / Accepted: 23 November 2020 / Published online: 26 November 2020

(C) Fondazione Società Italiana di Neurologia 2020

Sir,

On January 30, 2020, the World Health Organization (WHO) declared the emergence of a novel coronavirus (here named COVID-19), firstly reported few weeks earlier in Wuhan, Hubei Province of China, as a public health emergency of international concern. Since then, drastic countermeasures including shutdowns of public services have been implemented worldwide to reduce virus transmission: on March 9, 2020, the Italian Prime Minister imposed a national lockdown and ordered all hospitals to stop all non-urgent medical procedures, further aiming to release medical capacities for the management of COVID-19-infected patients.

Botulinum neurotoxin (BoNT) is a neurotoxic protein produced by the bacterium Clostridium botulinum, which is used in medical practice with a number of different indications including spasticity, dystonia, and chronic migraine [1]. As BoNT injections are usually given every 3 to 4 months, a large number of patients missed their scheduled treatment during the lockdown. Two initial reports described a significant worsening of this group of patients [2,3], highlighting the importance of BoNT treatment in the management of these chronic conditions. However, these results arose from uncontrolled studies [2,3]. We here report the results of a case-control study about the effects of the BoNT outpatient clinic shutdown on its patients and their health-related quality of life (HRQoL).

Soon after the ease of the lockdown restrictions in Italy on May 4, 2020, our center offered one BoNT extra-clinic per week to reschedule all willing patients who had missed their appointment because of the service shutdown (from here on named "cases"). Patients attending the BoNT service with their usual schedule in the same period served as controls.

Roberto Erro

rerro@unisa.it

1 Department of Medicine, Surgery and Dentistry "Scuola Medica Salernitana", University of Salerno, Via Allende, Baronissi, SA, Italy
All patients were asked to rate their perceived worsening on a visual analogue scale (VAS) ranging from 0 (no worsening) to 10 (worst imaginable worsening) in comparisons to the time they had the previous set of injections; similarly, they were asked to rate their attitude to repeat the injection during the lockdown period, would it have been possible. Finally, patients were administered a standardized, two-component, instrument to depict health status: the health state description (EQ-5D), which is measured in terms of five dimensions (namely, mobility, self-care, usual activities, pain/discomfort, and anxiety/depression) and the health state evaluation (EQ-VAS), through which the respondents evaluate their overall health status using a VAS ranging from 0 to 100. To allow comparisons between different BoNT formulations, doses of ONA-BoNT were standardized to ABOBoNT using a constant ratio of 1:2.5 international units (IU).

One-hundred thirty-seven patients agreed to participate: 94 cases and 43 controls. Cases had a mean delay of re-treatment of $73.61 \pm 26.54$ days. There were no differences between cases and controls in terms of age $(56.94 \pm 17.04$ vs $61.72 \pm$ 13.95 years; $t=1.60)$, sex distribution $(43 \mathrm{M} / 51 \mathrm{~F}$ vs $22 \mathrm{M} / 21 \mathrm{~F}$, $\left.\chi^{2}=0.34\right)$, disease duration $(11.38 \pm 3.40$ vs $12.34 \pm$ 2.79 years; $t=1.60$ ), underlying conditions (i.e., spasticity: $37.23 \%$ vs $32.55 \%$; dystonia: $40.42 \%$ vs $41.86 \%$; migraine: $10.63 \%$ vs $11.62 \%$; other: $11.70 \%$ vs $13.95 \%$; $\chi^{2}=2.47$ ), BoNT treatment duration $(5.04 \pm 5.62$ vs $4.7 \pm 6.11$ years; $t=-0.31)$, and BoNT doses $(659.54 \pm 197.11$ vs $673.49 \pm$ $193.76 \mathrm{IU} ; t=0.03$ ) (for all, $p>0.05$ ). BoNT doses did not differ between groups when stratifying for underlying conditions (data not shown). Cases reported a significant greater worsening of their condition than controls $(5.16 \pm 3.09$ vs $1.83 \pm 3.34$, respectively, $p<0.001$; Fig. 1 ). However, no differences were found in terms of attitude to repeat the injections, EQ-5D, and EQ-VAS (for all, $p>0.05$; Fig. 1). Additional analyses disclosed no significant differences in any of the gather variables within each group, when stratifying for either formulation of BoNT (i.e., ONA-BoNT vs ABOBoNT) or underlying conditions (data not shown). 
Fig. 1 Comparisons of the main variables between cases (red bars) and controls (blue bars). Data are expressed as mean \pm standard error; stars indicate statistical significance $(p<0.01)$
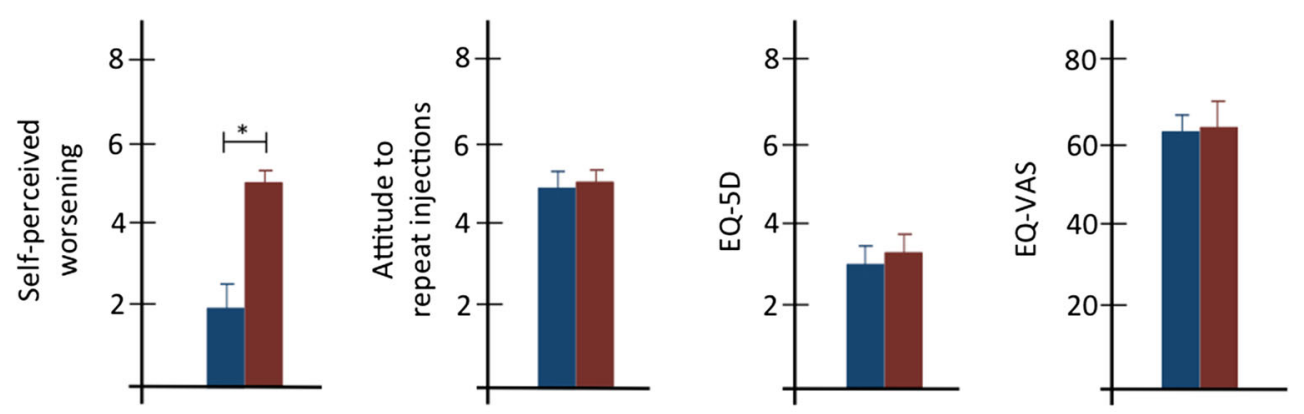

Usual schedule
Delayed injections
The COVID-19 pandemic has had unprecedented consequences on the medical care of many neurological patients. Whereas a huge effort has been put forward to implement telemedicine strategies to assure continuing care of these patients [4], those receiving BoNT injections have been basically excluded from any alternative options. Preliminary evidence suggested that significant clinical worsening occurred because of BoNT service shutdowns $[2,3]$. Whereas our results confirm this suggestion, they also show that this was not mirrored by a detrimental effect on HRQoL. The latter might seem contradicting the evidence stemming from randomized clinical trials for each of the BoNT indications. However, taking cervical dystonia for example since it accounted for the majority of patients in this study, it should be noted that a recent Cochrane meta-analysis has concluded that there is not enough evidence to definitively support a beneficial effect of BoNT injections on HRQoL [5]. In fact, at a close scrutiny, two out of three examined studies disclosed a significant improvement of the physical function domain for the active group, but not in social functioning when compared to placebo [5]. This example holds true for other BoNT indications and it should be stressed that there is an unacceptable lack of solid data about the long-term effects on HRQoL in patients receiving repeated BoNT injections. It might well be that over the years into the disease other factors, including the development of coping strategies and better acceptance of their condition, influence health status more than the injections themselves. In this regard, it is interesting to note that the attitude to be re-treated was similar in this study between cases and controls, further implying that patients attached the proper importance to BoNT therapy, perhaps in recognition of different health-related priorities and of the risk of exposure to COVID-19, particularly in a healthcare setting. This result further opens the question of what would be the best BoNT schedule to adopt in clinical practice and future research should clarify this issue, further considering both the pharmacoeconomic aspects of BoNT treatment and the indirect costs putatively associated with lengthening of the time interval between the injections.

We acknowledge the lack of validated scales to objectively measure baseline and follow-up disease severity. However, we also note that BoNT doses, which can be deemed as a proxy of disease burden, were not found to be different between cases and controls. It might be further argued that our HRQoL measures, despite being validated and largely utilized across different medical conditions, might be less sensitive in identifying significant changes compared to condition-specific instruments. Therefore, future studies adopting condition-specific scales are warranted. It should be however stressed that one of the unique advantages of using such instruments as EQ-5D and EQ-VAS consists in comparing and generalizing the results across different conditions.

While it is undoubtful that novel strategies to ensure continuing care of chronic neurological patients needing regular procedures/infusions have to be carefully thought out as this virus continues to spread, it is equally imperative to exploit this dramatic experience as food for thought about the standard care we deliver and how we can do better.

\section{Authors' contributions}

(1) $\mathrm{A}=$ conception; $\mathrm{B}=$ data collection; $\mathrm{C}=$ analysis

(2) $\mathrm{A}=$ writing the first draft; $\mathrm{B}=$ revising the manuscript

RE: $1 \mathrm{~A}-\mathrm{C}, 2 \mathrm{~A}, \mathrm{~B}$

SS: $1 \mathrm{~B}, 1 \mathrm{C}, 2 \mathrm{~B}$

MR: 1B, 1C, 2B

MP: 1B, 2B

PB: $1 \mathrm{C}, 2 \mathrm{~B}$

Data availability Data are available upon reasonable request to the corresponding author (rerro@unisa.it).

\section{Compliance with ethical standards}

Conflict of interest The authors declare that they have no conflict of interest.

Ethical approval This study was approved by the local ethics committee and has been performed in accordance with the ethical standards laid down in the 1964 Declaration of Helsinki and its later amendments.

Informed consent All subjects provided a written consent form to participate.

Consent for publication All subjects provided a written consent form to publish their anonymized data.

Code availability Not applicable 


\section{References}

1. Dressler D (2016) Botulinum toxin drugs: brief history and outlook. J Neural Transm (Vienna) 123(3):277-279

2. Ali A (2020) Delay in onabotulinumtoxinA treatment during the COVID-19 pandemic-perspectives from a virus hotspot. Headache. 60(6):1183-1186

3. Dressler D, Adib SF (2020) Botulinum toxin therapy in the SARSCoV-2 pandemic: patient perceptions from a German cohort. J Neural Transm (Vienna) 127(9):1271-1274
4. Mulroy E, Menozzi E, Lees AJ, Lynch T, Lang AE, Bhatia KP (2020) Telemedicine in movement disorders: Leçons du coronavirus disease 2019. Mov Disord 35:1893-1896. https://doi.org/10.1002/ mds. 28297

5. Castelão M, Marques RE, Duarte GS, Rodrigues FB, Ferreira J, Sampaio C, Moore AP, Costa J (2017) Botulinum toxin type A therapy for cervical dystonia. Cochrane Database Syst Rev 12(12): CD003633

Publisher's note Springer Nature remains neutral with regard to jurisdictional claims in published maps and institutional affiliations. 